\title{
Correlation between Occurrence of Pearl Pulsations and Interplanetary Magnetic Field Sector Boundaries
}

\author{
R. L. McPherron AND S. H. WARD \\ Space Sciences Laboratory, University of California, Berkeley
}

\begin{abstract}
A significant correlation between the occurrence of pearl pulsations at College, Alaska, and interplanetary magnetic field sector boundaries has been observed. Pearl pulsation data published by the Geophysical Institute, University of Alaska, and sector data published by Wilcox and Ness have been used to show the following: pearl pulsations are more likely to occur on the day a sector boundary passes the earth than in the middle of a sector. Pearl pulsations occur with maximum probability near 1500 local time on all sector days but are restricted almost entirely to local afternoon in the middle of a sector. On the average, fewer pearl pulsations are observed during magnetically disturbed times than quiet times, but in the afternoon pearl occurrence is enhanced by geomagnetic disturbance. Pearls begin and end later in the day as the level of magnetic activity increases.
\end{abstract}

\section{INTRODUCTION}

Wilcox and Ness [1965] have demonstrated that at certain times the interplanetary magnetic field can be characterized by a well-defined sector structure such as that shown in the top half of Figure 1. Magnetic field lines from the sun are pulled outward by the solar wind and wrapped into spirals by the solar rotation. Alternate regions of the sun, and hence sections of interplanetary space, are found to have weak magnetic fields of predominantly one polarity. Wilcox and Ness also find that various parameters of the solar wind as well as geomagnetic activity are well organized by the sector boundaries. This is interpreted to mean that these parameters are systematic functions of time within the solar sector. Results they have obtained are shown in the bottom portion of the figure. As indicated in the bottom right curve, geomagnetic activity as measured by sum $K_{p}$ is a maximum on the second day of the 7- to 8-day sectors.

As shown in this paper, pearl pulsations, the most outstanding phenomenon in the $\mathrm{Pc}$ 1 band, are also correlated with the interplanetary field sectors. We have used data on the occurrence of pearls observed at College, Alaska, which were published in the High Latitude Geophysical Data series of the University of Alaska [Hessler, 1964]. Data on the sectors were obtained from Wilcox and Ness (personal communication).

\section{EXPERIMENTAL RESULTS}

The total number of occurrences of pearls as a function of day in the solar rotation interval for the first six months of 1964 are plotted
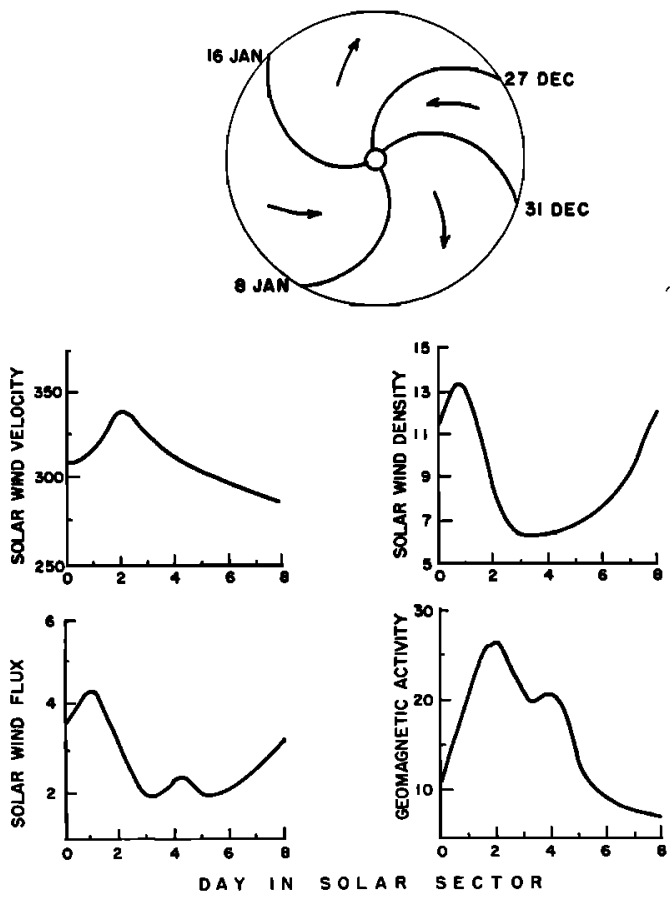

Fig. 1. Interplanetary magnetic field sectors observed by Wilcox and Ness during December 1963 and January 1964. Geophysical parameters organized by sector structure. 


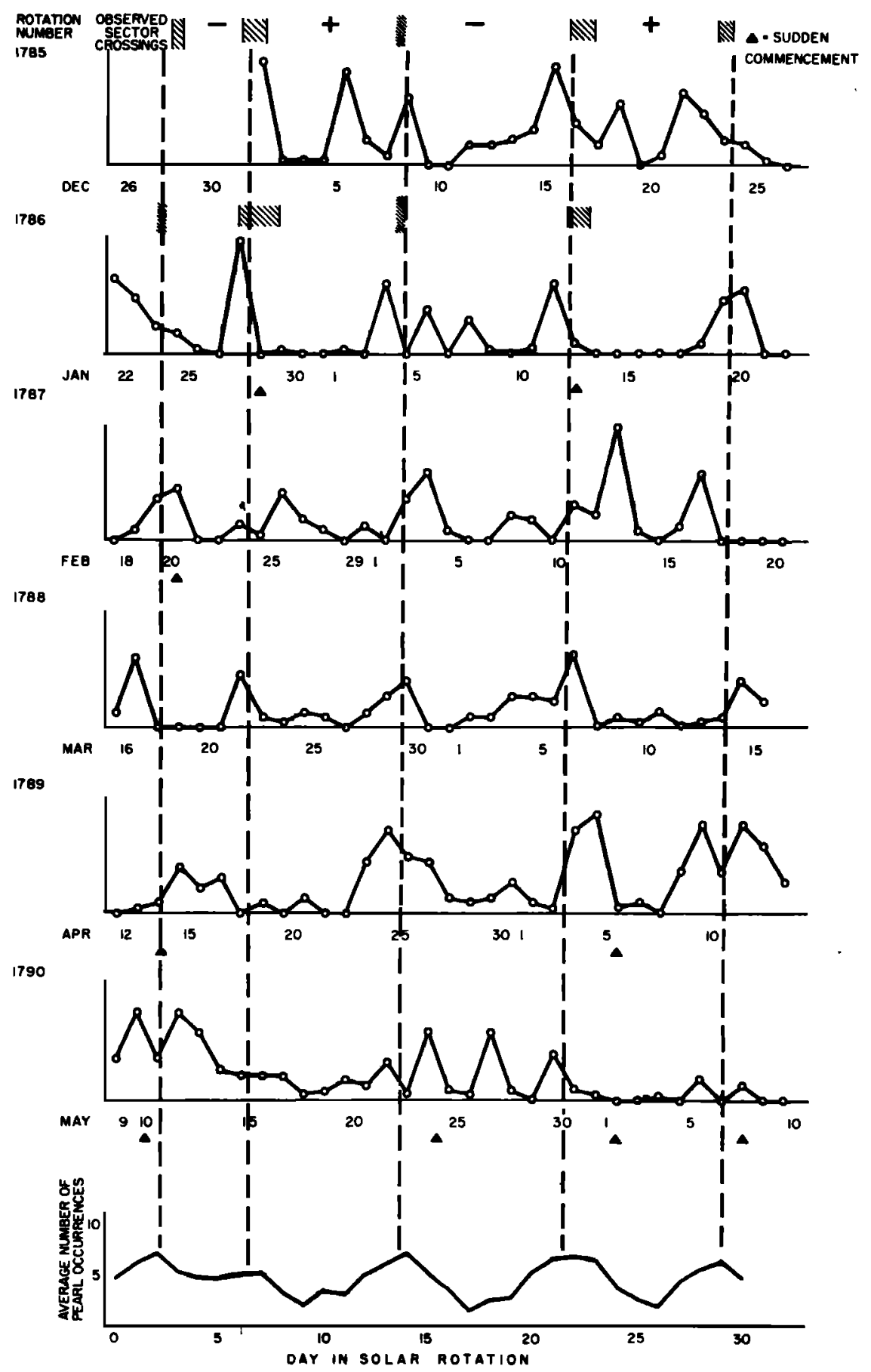

Fig. 2. Number of half-hour intervals per day during which pearl pulsations were observed at College, Alaska, for first six solar rotation intervals of 1964. Observed and extrapolated sector boundary crossings are indicated. The average number of occurrences obtained by superposed epoch analysis is also shown. 
in Figure 2. The times at which the boundaries of the sectors were observed to pass the earth are shown hatched in the first two rotation intervals. Extrapolation of these crossing for an additional four intervals is shown by the dashed vertical lines. Following one of these lines vertically, one observes a slight tendency for pearls to occur near a sector boundary. However, by performing a superposed epoch analysis and smoothing the resulting curve by taking a 3-day running average to allow for uncertainties in the time of sector crossing, the result shown at the bottom is obtained. It will be noted that every extrapolated boundary crossing corresponds to a peak in the occurrence of pearls. In particular, pearls are twice as probable on the day of a sector crossing as in the middle of a sector.

To examine the validity of the superposed epoch analysis, it was repeated on a basis of various recurrence intervals from 25 to 30 days. The smoothed results are shown in Figure 3 . The curve corresponding to 27 days (the synodic rotation period of low-latitude regions on the sun) stands out from the others in several respects. The fluctuations from the mean are somewhat larger and considerably more regular than is true for other assumed intervals. The maxima of the curve correspond to the sector crossings. However, the 27-day curve does not stand out from the others as distinctly as one might hope. An examination of the original data in Figure 2 suggests that this is mainly a consequence of the small amount of data available for the superposed epoch analysis, combined with considerable irregularity in the phenomenon being studied.

By taking fourteen successive 7- to 8-day sectors and superposing each of them, it is possible to improve the statistics considerably. In addition, it becomes possible to examine the effects of parameters other than day within a solar sector. In Figure 4 are considered the effects of local time as well as sector day. At the top right is plotted the probability of occurrence of pearls as a function of local time. The most probable time of occurrence is seen to be the middle of the afternoon. On the bottom left is the probability of occurrence of pearls as a function of day in solar sector. The enhanced probability of occurrence on the days of the sector crossings is evident. On the bot-

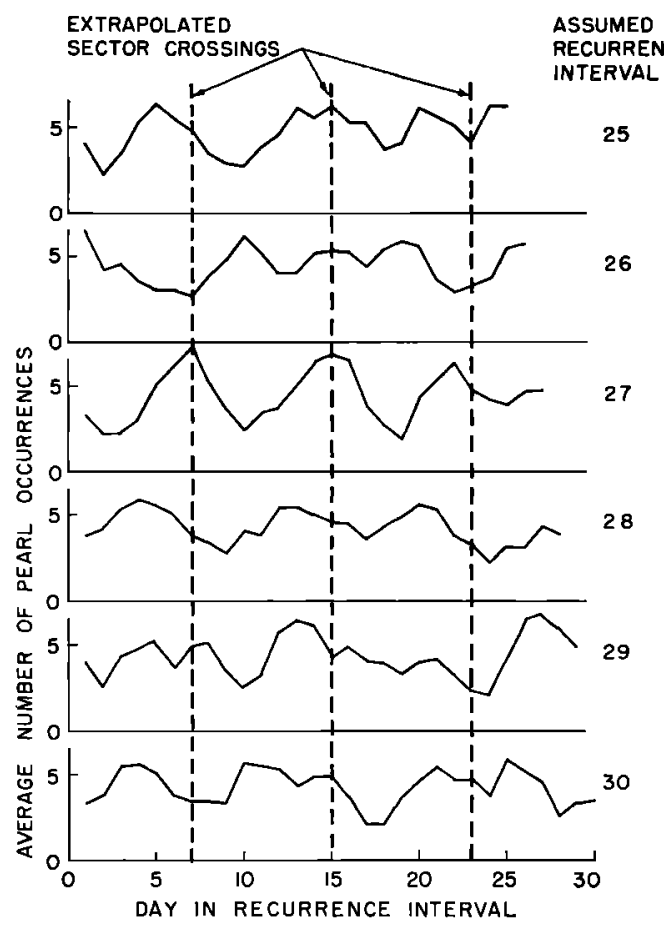

Fig. 3. Superposed epoch analysis of pearl occurrence for various assumed recurrence intervals.

tom right of the figure are drawn contours of constant probability of pearl occurrence as a function of both sector day and local time. Two prominent features are apparent from an examination of the contour diagram. Pearls are likely to occur at almost any hour on the day of a sector crossing. In the middle of a sector, however, they are limited to times between local noon and sunset.

As mentioned above, the data plotted in these and succeeding figures have been smoothed in both variables by taking three-point running averages. This process corresponds numerically to passing the data through low pass filters with time constants of the order 2 hours for local time and 2 days for solar sector time. Application of this process effectively eliminates rapid fluctuation due to insufficient statistics. In addition, it smooths out the effects of uncertainties in the data, as for example, in the extrapolation of the day of a sector crossing.

Figure 4 does not take into account the effect of the level of geomagnetic activity on pearl occurrence as previously reported by 


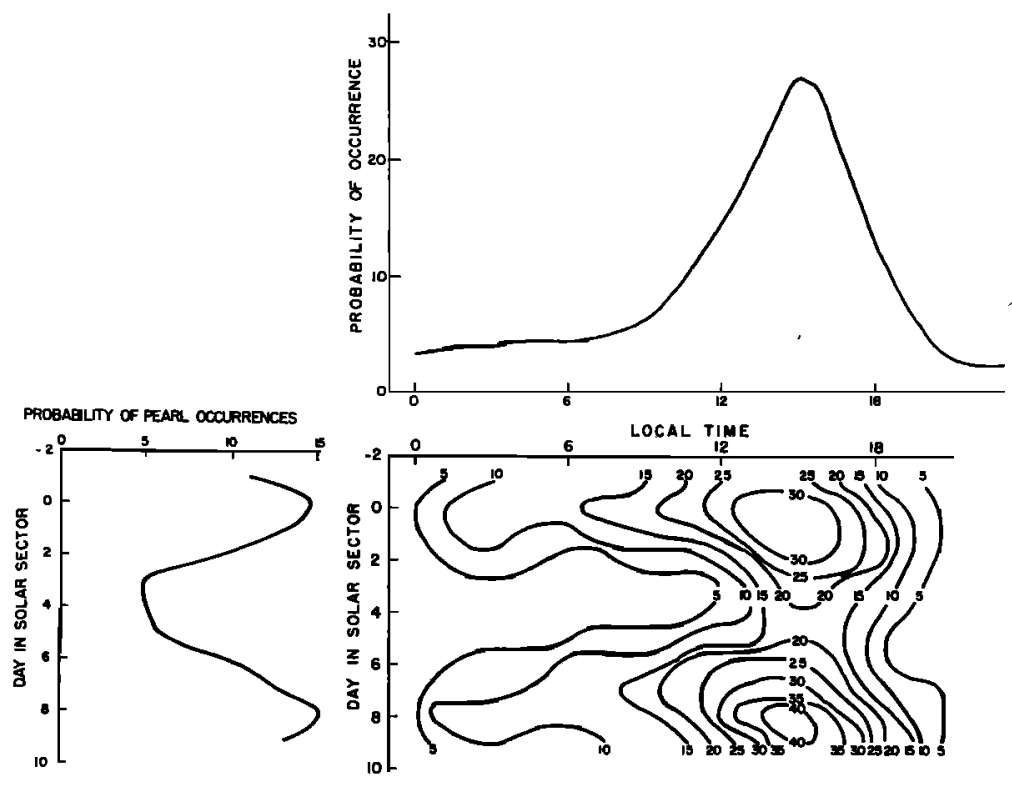

Fig. 4. Smoothed probability of pearl occurrence as a function of both day in solar sector and local time.

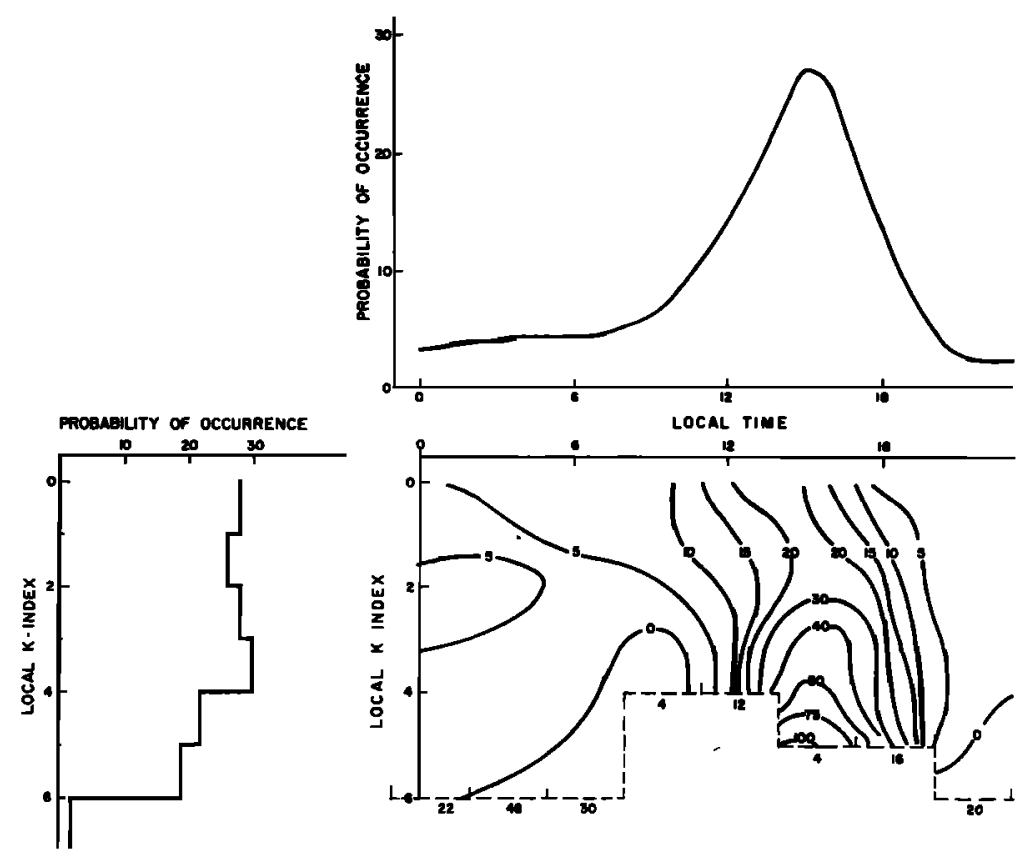

Fig. 5. Smoothed probability of pearl occurrence as a function of both local $K$ index and local time. 
McPherron and Ward [1965]. In Figure 5, the probability of occurrence of pearls as a function of both local time and local magnetic activity is presented. In the top right portion of the figure is repeated the average diurnal dependence of pearl occurrence. In the lower left is plotted the probability of occurrence as a function of the local magnetic $K$ index. Through $K=3$, pearl occurrence appears to be independent of $K$. Above $K=3$, the occurrence probability appears to decrease with increasing $K$. In the bottom right of the figure are plotted contours of constant probability of pearl occurrence as a function of both local time and magnetic $K$ index. The functional dependence on these two parameters is seen to be fairly complicated. Throughout most of the day the occurrence probability decreases with increasing magnetic activity as suggested by the average result. However, during the afternoon pearl occurrence becomes more probable with increasing magnetic activity. In fact, for each increment in $K$, pearls begin and end later in the day and are enhanced in their probability of occurrence.

From the preceding two figures it is evident that the level of geomagnetic activity is more significant in determining the diurnal dependence of pearls than is sector structure. As noted in Figure 1, the level of geomagnetic activity is itself a function of the sector structure. However, on any particular sector day there is some spread about the average in the level of geomagnetic activity from sector to sector. One can make use of this to make an additional separation of the sector data into various levels of magnetic activity. In Figure 6 are plotted contours of constant probability of occurrence of pearls as a function of local time and sector day for three different levels of the magnetic $K_{\mathrm{p}}$ index. The general features discussed above are all evident: enhanced probability on the day of sector crossings, reduced probability in the middle of a sector, and restriction to the afternoon and enhancement of occurrence as the level of magnetic activity increases. One new feature appears, suggesting that pearls are more likely to occur in the middle of a sector if geomagnetic activity is very low.

Several other characteristics of pearl pulsations, besides occurrence were examined as a
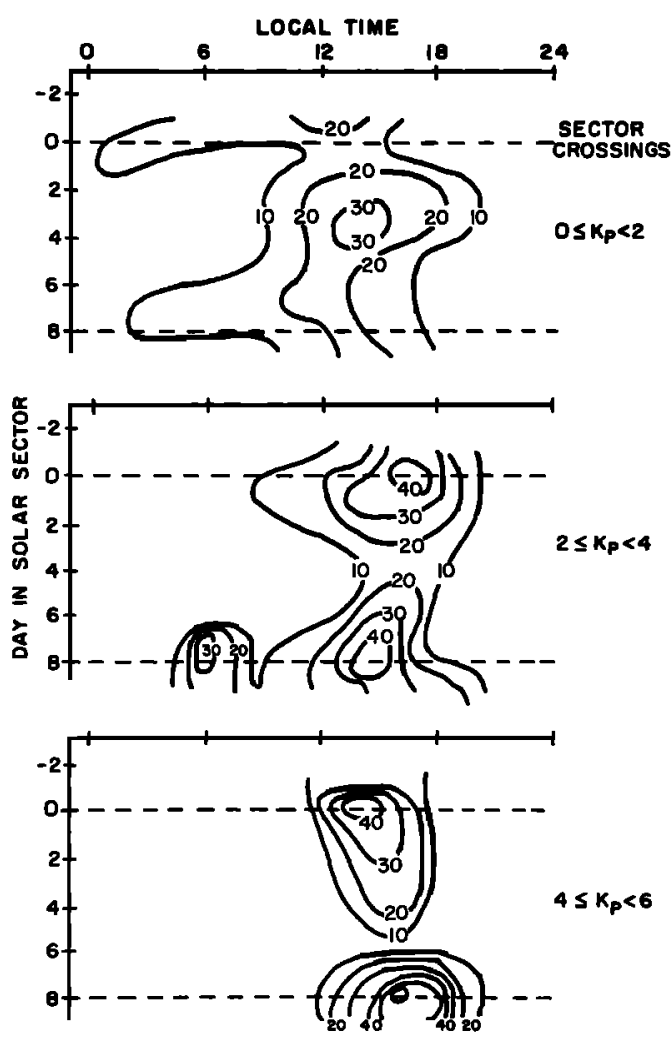

Fig. 6. Smoothed probability of pearl occurrence as a function of three significant variables: local time, day in solar sector, and level of worldwide magnetic activity.

function of day in the sector structure. Amplitude, frequency, and pearl spacing do not appear to depend on the sector structure.

Pearl occurrence data for the last six solar rotation intervals in 1964 were also examined by the superposed epoch technique. The particular sector structure observed by Wilcox and Ness [1965] and shown to be correlated with pearl pulsations in this paper apparently did not persist for the remainder of the year. Examination of the pearl occurrence data, as well as charts of the magnetic character figure $C 9$ suggest that the sector structure altered rapidly during August and September 1964.

\section{Discussion}

In an attempt to understand these results concerning pearl pulsations, we must re-examine Figure 1. We note several extrema of the solar wind parameters may possibly be corre- 
lated with the occurrence of pearl pulsations. First, there are three important properties of the solar wind on the day of a solar sector crossing: (1) the interplanetary magnetic field abruptly reverses direction; (2) the solar wind velocity is at a minimum; (3) geomagnetic activity is at a minimum. On the other hand, the middle of $a^{\prime}$ sector is characterized by minima in the solar wind density and particle flux.

Present ideas on the origin of pearl pulsations as discussed by Jacobs and Watanabe [1964] and Obayashi [1965] hold that a hydromagnetic pulse is triggered by some means, somewhere along a magnetic field line. The pulse is guided along the line and reflected at the end by the upper limits of the ionosphere. In passing through the equatorial plane, it encounters trapped protons and may be amplified by a cyclotron instability [Cornwall, 1965; Jacobs and Watanabe, 1965], the instability arising as a consequence of an anisotropic pitch-angle distribution in the trapped protons, the cause of which is not known.

One of the difficulties in interpreting some of the preceding observations in terms of this model is that the magnetic $K$ index used is not a fundamental parameter. It depends upon various solar wind characteristics, such as magnitude of solar wind velocity, direction or component of interplanetary field perpendicular to the ecliptic, degree of turbulence in the solar wind, and perhaps other parameters as well.

In general, it might be expected that the effects of increased magnetic disturbance within the magnetosphere would be to destroy anisotropic pitch-angle distributions and disrupt the guiding of the hydromagnetic pulses, resulting in a lower probability of pearl occurrence as observed. On the other hand, if the anisotropy persists despite the disturbance at some hour angle, then magnetic disturbance may be advantageous inasmuch as it could provide the triggering pulse that is then amplified to produce the pearl pulsations. Such behavior could possibly account for the enhanced probability of pearl occurrence at certain local times.

The increase of occurrence probability on the day of sector crossings is apparently related to more favorable conditions for establishing the necessary pitch-angle anisotropy. Possibly, reversal of the interplanetary field direction causes rearrangement of particle populations, producing the needed anisotropy for a short time. It is perhaps significant in this regard that the 1500 local-time maximum corresponds roughly to the hour angle at which spiral lines in the corotating structure would first begin interacting with the magnetosphere.

The minimum occurrence of pearls in the middle of a sector may be related to the minimum in the solar wind proton density, which also occurs at this time.

Acknowledgment. This work was supported in part by the National Science Foundation under grant 5331.

\section{References}

Cornwall, J. M., Cyclotron instabilities and electromagnetic emission in the ultra low frequency and very low frequency ranges, J. Geophys. Res., 70, 61-69, 1965.

Hessler, V. P., editor, High latitude geophysical data, January-July, 1964, Geophys. Inst., Univ. of Alaska, Rept. UAG-C (\$5-86), 1964.

Jacobs, J. A., and T. Watanabe, Micropulsation whistlers, J. Atmospheric Terrest. Phys., 26, 825-829, 1964.

Jacobs, J. A., and T. Watanabe, Amplification of hydromagnetic waves in the magnetosphere by a cyclotron instability process with applications to the theory of hydromagnetic whistlers, Boeing Sci. Res. Lab., Rept. D1-82-0398, January 1965.

McPherron, R. L., and S. H. Ward, Auroral-zone pearl pulsations, J. Geophys. Res., 70, 5867$5882,1965$.

Obayashi, T., Hydromagnetic whistlers, J. Geophys. Res., 70, 1069-1078, 1965.

Wilcox, J. M., and N. F. Ness, Quasi-stationary co-rotating structure in the interplanetary medium, J. Geophys. Res., 70, 5793-5805, 1965.

(Received August 12, 1966.) 